INPLASY

PROTOCOL

To cite: Wu et al. Efficacy of electroacupuncture for the treatment of asthenozoospermia: a protocol for systematic review and meta-analysis. Inplasy protocol 2020100071. doi:

10.37766/inplasy2020.10.0071

Received: 20 October 2020

Published: 20 October 2020

Corresponding author:

Xinhui Wu

775829138@qq.com

Author Affiliation:

Hospital of Chengdu

University of TCM

Support: 1BZL09.

Review Stage at time of this submission: The review has not yet started.

Conflicts of interest:

The authors report no conflicts of interest in this work.

\section{Efficacy of electroacupuncture for the treatment of asthenozoospermia: a protocol for systematic review and meta-analysis}

Wu, XH¹; Chen, DA2; Zhou, YX33 Xia, T4.

Review question / Objective: The purpose of this study is to further evaluate the effectiveness and safety of EA in the treatment of asthenospermia. The results will provide urologists and andrologists with clinical surgery decisions.

Condition being studied: Infertility has affected millions of couples aged 15-44 worldwide. Recently, some studies suggest that abnormal semen quality is the main cause of male infertility and asthenozoospermia accounts for $19 \%$ of the infertility of men. The situation has brought a huge burden to the patient with asthenozoospermia and society. Acupuncture is a part of traditional Chinese medicine. Electroacupuncture has gained in popularity. Although a positive effect of manual acupuncture and electroacupuncture on sperm parameters has been documented in several studies, there still a lack of more solid evidence. We hope to provide a convincing study for EA.

INPLASY registration number: This protocol was registered with the International Platform of Registered Systematic Review and Meta-Analysis Protocols (INPLASY) on 20 October 2020 and was last updated on 20 October 2020 (registration number INPLASY2020100071).

\section{INTRODUCTION}

Review question / Objective: The purpose of this study is to further evaluate the effectiveness and safety of EA in the treatment of asthenospermia. The results will provide urologists and andrologists with clinical surgery decisions.
Condition being studied: Infertility has affected millions of couples aged 15-44 worldwide. Recently, some studies suggest that abnormal semen quality is the main cause of male infertility and asthenozoospermia accounts for $19 \%$ of the infertility of men. The situation has brought a huge burden to the patient with asthenozoospermia and society. 
Acupuncture is a part of traditional Chinese medicine. Electroacupuncture has gained in popularity. Although a positive effect of manual acupuncture and electroacupuncture on sperm parameters has been documented in several studies, there still a lack of more solid evidence. We hope to provide a convincing study for EA.

\section{METHODS}

Participant or population: Inclusion criteria: -Diagnosed as asthenospermia based on WHO reference values. The diagnosis was confirmed by at least two consecutive semen analyses performed on ejaculates collected after 3-7 days of sexual abstinence. Exclusion criteria: Patient with ejaculatory dysfunction. Patients with a medical history of risk factors for infertility (eg, prior vasectomy, orchidopexy, or varicocele). Patients with immunerelated infertility. Patients with occupational exposure to agents suspected to impact male reproduction. -Patients with a history of previous infertility treatment (eg, hormonal therapy). -Patients with genetic disease.

Intervention: The patients in the treatment group received EA.

Comparator: The control group could gain hormonal therapy, antioxidant therapy, trace elements, energy support therapy, or guideline-recommended conventional treatment.

Study designs to be included: All the RCTs of EA for patients with asthenospermia will be included without publication status restriction or writing language letters to editors, review articles, case reports, conference abstracts, cross-sectional studies, and all observational studies will be excluded.

Eligibility criteria: All the RCTs of EA for patients with asthenospermia will be included without publication status restriction or writing language letters to editors, review articles, case reports, conference abstracts, cross-sectional studies, and all observational studies will be excluded.

Information sources: The electronic databases of MEDLINE, PubMed, Web of Science, EMBASE, Cochrane Library, Clinicaltrials. org, China National Knowledge Infrastructure Database (CNKI), Wan fang Database, China Biology Medicine Database (CBM), VIP Science Technology Periodical Database, Chinese Clinical Trial Registry will be retrieved. They will be searched until October 2021 to recognize related studies. The search strategy that will be run in the PubMed and adjusted to fit the other database.

Main outcome(s): 1) Sperm motility.

\section{Additional outcome(s): 1) Sperm DNA fragmentation index (DFI).}

Quality assessment / Risk of bias analysis: According to pre-defined eligibility criteria, importing the literature retrieved to the Endnote X8 and eliminate the duplicate data. The software will be used to filter duplicate documents first, and then the studies which don't meet the inclusion criteria will be removed. If the studies appear to meet the inclusion criteria or there is any uncertainty based on the information provided in the title and abstract, full texts will be obtained for further assessment. Further detailed screening and data extraction of the documents will be performed simultaneously by two professionally trained reviewers. When necessary, the original study author will be contacted for judgment. Disagreements will be resolved by discussion or taking the expert (DAC) for arbitration. The number and reasons for excluding trials will be recorded in detail.

Strategy of data synthesis: The RevMan5.3 software will be used to conduct the metaanalysis (If feasible). Descriptive analysis or systematic narrative synthesis will be performed to summarize and explain the characteristics and findings of the included studies and provide the information in the texts and tables. For dichotomous data (e.g., effective and ineffective), we will 
calculate risk ratio (RR) and $95 \%$ confidence intervals (Cls). For continuous data, which will be pooled as mean difference (MD).

Subgroup analysis: If necessary, we will identify the source of heterogeneity through subgroup analysis and manage the heterogeneity: 1) the duration and severity of asthenospermia. 2) demographic characteristics of the patients: age, marital and family status, region, race. 4) therapy time 3) follow-up time.

Sensibility analysis: Sensitivity analysis will be used to test the reliability and stability of the meta-analysis results, and to assess the source of heterogeneity. We will compare the results before and after by excluding trials with a high risk of bias or eliminating each study individually one study each time and then pooling the remaining studies.

Language: English.

Country(ies) involved: China.

Keywords: electroacupuncture; asthenozoospermia; protocol; systematic review

Contributions of each author:

Author 1 - Xinhui Wu contributed to the development of the selection criteria, and the risk of bias assessment strategy.

Author 2 - Diang Chen contributed to the development of the selection criteria, and the risk of bias assessment strategy.

Author 3 - Yexin Zhou contributed to the development of the selection criteria, and the risk of bias assessment strategy.

Author 4 - Ting Xia contributed to the development of the selection criteria, and the risk of bias assessment strategy. 